\title{
Uncommon brain metastases from endometrial adenocarcinoma, 2 Case reports
}

\author{
Yuan Frank Shan*, Buqing Liang, Frank Harris and Jason H Huang \\ Department of Neurosurgery, Texas A\&M HSC College of Medicine, USA \\ *Corresponding author: Yuan Frank Shan, Department of Neurosurgery, Baylor Scott \& White Health, 2401 S 31st Street, Temple, TX, \\ USA \\ To Cite This Article: Yuan Frank Shan. Uncommon brain metastases from endometrial adenocarcinoma, 2 Case reports. 2020 - 9(4). AJBSR. \\ MS.ID.001418. DOI: 10.34297/AJBSR.2020.09.001418.
}

Received: 眥 May 23, 2020; Published: 觜 July 15, 2020

\section{Introduction}

Brain tumor accounts for only about $1 \%$ of human body neoplasms, among those brain tumors, metastatic tumors take up a significant percentage, probably due to the advance treatment which makes patients' survival longer than before. Brain metastases, in North America, usually come from lung cancers, breast cancers and

malignant melanomas, probably due to their relatively closer locations to the brain by anatomy. Long-distance metastases from organs below diaphragm/intra-abdominal organs are uncommon, except for renal cell carcinoma, which most often metastasizes to the cerebellum. Here we report 2 cases of endometrial carcinomas metastasized to the brain.

Case 1

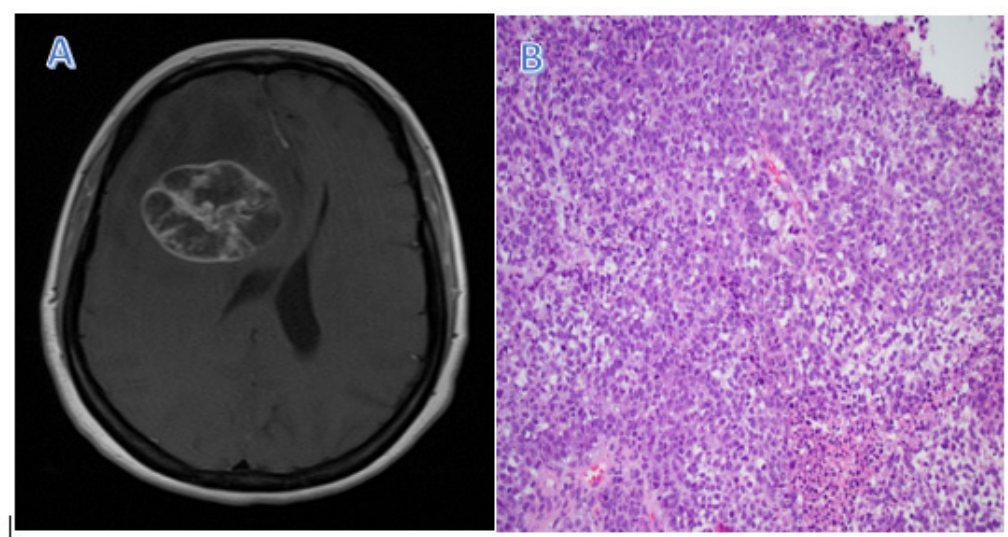

Figure 1: MRI scan reveals a cystic mass in the right frontal lobe with mass effect of midline shift and heterogeneous enhancing (A). Microscopic examination reveals the tumor in a solid pattern with focal clear cytoplasm, indicating a high-grade endometrial adenocarcinoma (H\&E, X200) (B).

Patient is a 36-year-old otherwise healthy female with a past medical history of endometrial cancer (endometrioid carcinoma with squamous differentiation) status post hysterectomy 9 months ago, with chemotherapy and radiation therapy who presented with intermittent headache accompanied with nausea for three weeks. A brain MRI with and without contrast was obtained which revealed a right frontal intra-axial cystic contrast-enhancing lesion about
$5.3 \mathrm{~cm} \times 4.3 \mathrm{~cm} \times 4.3 \mathrm{~cm}$. Her physical examination was non-focal and lab workup was unremarkable. The patient underwent right frontal craniotomy and lesion resection without complications. Post-operative brain MRI with and without contrast on postoperative day 1 indicated gross total resection of the lesion and pathology report returned as metastatic high-grade endometrial adenocarcinoma (Figure 1). 


\section{Case 2}

Patient is an 87-year-old female with a past medical history of hypertension, GERD, hypercholesteremia, hypothyroidism, endometrial cancer (papillary serous adenocarcinoma) status post hysterectomy six years ago status post chemotherapy who presented with sudden onset of headache accompanied with nausea and vomiting for one day. Workup revealed a right cerebellar cystic contrast-enhancing lesion about $4.2 \mathrm{~cm} \mathrm{x} 4 \mathrm{~cm}$ x
$3.1 \mathrm{~cm}$ on brain MRI with and without contrast (Fig. Her physical examination was non-focal, and labs were unremarkable. She underwent right suboccipital craniotomy and resection of the lesion without complications. Post-operative brain MRI with and without contrast on post-operative day 1 revealed a gross total resection and pathology report returned as a poorly differentiated metastatic adenocarcinoma. By immunohistochemical staining reveals the tumor cells are positive for CK-7, P53, PAX-8 and ER/PR, consistent with an endometrial primary (Figure 2).
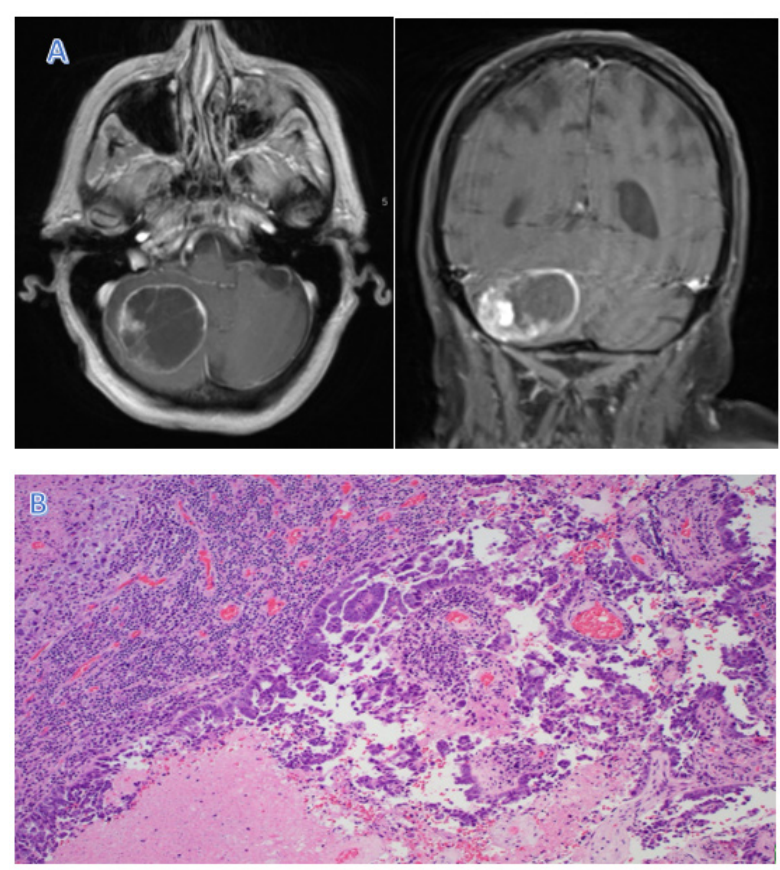

Figure 2: MRI shows a cystic lesion in the right cerebellum with peripheral rim enhancing and an intracystic mural nodule (A). Microscopic features show the tumor cells with focal papillary architecture and extensive necrosis. With the help of immunohistochemical stains, this tumor is consistent with endometrial origin (H\&E, X400) (B).

\section{Discussion}

Metastasis is a complex genetic and biochemical process commonly seen in malignant neoplasms. It not only characterizes the malignant nature of the tumor, but also is the major cause of cancer death. Metastatic brain tumors are primarily from lung, breast cancers and melanoma, due to their aggressive behavior and relatively close anatomic relations. Meanwhile, a metastatic brain lesion from the organs below the diaphragm/intraabdominal organs are very rare. Most of this type of remote metastasis need to set up a first metastatic site (usually lung, or liver) and then metastasize to the brain, except renal cell carcinoma. For unknown reasons, renal cell carcinoma has a preference for cerebellum, and rarely reaches the cerebral hemispheres. Brain metastasis usually locates at grey and white matter junction as a result of the rich blood supply in this region. As the metastatic brain tumors from cervical squamous carcinomas we reported [1], those brain metastases from gynecological neoplasms are very rare, sometimes requiring extensive immunohistochemical stains to search for the primary tumor by the pathologists. For the treatment of brain metastasis, gross total resection is the choice. If any residual tumor is identified on the post-operative MRI scan, radiation and chemotherapy including selected target treatment should be considered. Furthermore, meticulous review of the patient's history is very helpful to solve the puzzles in the clinical setting.

\section{Reference}

1. Setoodeh R, Hakam A, Shan Y (2012) Cerebral metastasis of cervical cancer, report of two cases and review of the literature. Int J Clin Exp Pathol 5(7): 710-714. 